
allemande

48-1 | 2016

Les expériences coloniales allemandes : échanges, transferts, circulations

\title{
Dominique BOUREL, Martin Buber. Sentinelle de
}

l'humanité

Paris, Albin Michel, 2015

Sonia Goldblum

\section{(2) OpenEdition}

\section{Journals}

Édition électronique

URL : https://journals.openedition.org/allemagne/350

DOI : 10.4000/allemagne.350

ISSN : 2605-7913

Éditeur

Société d'études allemandes

Édition imprimée

Date de publication : 15 juin 2016

Pagination : 227-229

ISSN : 0035-0974

Référence électronique

Sonia Goldblum, "Dominique boureL, Martin Buber. Sentinelle de l'humanité », Revue d'Allemagne et des pays de langue allemande [En ligne], 48-1 | 2016, mis en ligne le 13 décembre 2017, consulté le 28 mai 2021. URL : http://journals.openedition.org/allemagne/350 ; DOI : https://doi.org/10.4000/allemagne. 350 
Freitag étudie pour sa part les "représentations de l'identité juive dans l'œuvre de Gertrud Kolmar» (1894-1943). Elle évoque les caractéristiques d'une écriture marquée par le non-dit et le sentiment de l'altérité et de l'exclusion, à la recherche d'un judaïsme authentique au-delà de l'assimilation.

La troisième et dernière partie met l'accent sur le paradigme urbain, pris comme un angle d'approche particulièrement pertinent pour traiter des identités juives. Joachim Schlör, dans un article intitulé "Les Juifs et la grande ville», évoque l'intérêt porté par les études juives du monde entier au fait urbain, qui suggère un lien naturel entre les Juifs et la vie urbaine. II reprend ici les idées centrales de sa thèse d'habilitation intitulée Das Ich der Stadt (Göttingen, Vandenhoeck \& Ruprecht, 2005). II part de ce constat pour appeler de ses voux un changement de paradigme dans les études juives, pour travailler non plus en termes de temps mais en termes d'espace. Laurence Guillon traite de la vie juive à Berlin à partir de 1945, dont elle évoque à la fois les différentes étapes et les lieux caractéristiques de son émergence, ainsi que les «vecteurs de pérennité». Elle aussi reprend certains des points centraux de sa thèse de doctorat, publiée sous le titre La vie juive à Berlin après 1945. Entre Est et Ouest (Paris, CNRS, 2012). Dans ce contexte, la catégorie de la "symbiose négative», qui reprend celle de "symbiose judéo-allemande», critiquée en son temps par Gershom Scholem, joue un rôle important. Pour la période d'après 1945, la "symbiose négative» qualifie le lien qui perdure entre Juifs et Allemands sous le signe de la Shoah et du rapport entre victimes et criminels nazis. Le dernier article traite également de cette catégorie et évoque le renouveau de la vie juive en Allemagne après la réunification, caractérisée par la «réappropriation de repères identitaires culturels et religieux juifs, étroitement liés à la Wende». Cette renaissance est tenue à la fois par l'immigration de Juifs venus de l'ex-URSS et par l'attraction exercée par la ville de Berlin sur les Israéliens depuis les années 2000.

Cet ouvrage livre un panorama de la recherche française actuelle sur les Juifs dans l'espace germanophone, et ce d'autant mieux que les articles des plus jeunes des contributeurs portent sur leur sujet de thèse et en donnent pour ainsi dire un condensé. En somme cet ouvrage rend compte de la très grande richesse et du dynamisme de la recherche française dans ce domaine.

À bien des égards, cette publication constitue le pendant d'un autre ouvrage collectif, également publié en 2014 aux Presses universitaires de Rennes, sous la direction de Daniel Baric, de Tristan Coignard et de Gaëlle Vassogne, et qui portait sur les Identités juives en Europe centrale. Des Lumières à l'entre-deux guerres.

Sonia Goldblum

Dominique Bourel, Martin Buber. Sentinelle de l'humanité, Paris, Albin Michel, $828 p$.

Dans le domaine de l'étude et de l'histoire du judaïsme allemand, Dominique Bourel est pour ainsi dire une figure tutélaire et son livre sur Martin Buber était très attendu depuis de nombreuses années par la communauté scientifique. Il est le résultat d'un travail d'archive minutieux et de grande ampleur, dont Bourel rend compte au début de la bibliographie (p. 792-793). Il signale notamment l'importance de la correspondance de Buber, qui comprend cinquante mille lettres, pour l'élaboration de cette étude. 
L'accueil dont cette biographie a bénéficié depuis sa parution en novembre 2015 est à la mesure de l'importance de l'ouvrage.

En introduction, l'auteur compare lui-même son livre avec sa monumentale biographie de Moses Mendelssohn, parue en 2004 sous le titre Moses Mendelssohn. La naissance du judaïsme moderne (Paris, Gallimard, 2004). Dominique Bourel a coutume de dire que la traduction de la Bible de Mendelssohn et celle de Martin Buber et de Franz Rosenzweig, entamée en 1925, et que Buber poursuivra seul après le décès de Rosenzweig en 1929, marquent deux bornes dans l'histoire du judaïsme allemand (ibid., p. 355), la première accompagnant l'émancipation des Juifs allemands en leur apprenant cette langue, écrite à cet effet en caractères hébraïques, la seconde leur permettant de renouer avec une tradition juive dont ils s'éloignent de plus en plus, en leur faisant entendre les sonorités de l'hébreu à travers l'allemand. Ces deux livres contribuent donc à rendre compte et à contextualiser deux moments capitaux de l'histoire des Juifs. On reconnaît également dans la structure du livre la passion de Dominique Bourel pour l'aventure urbaine et pour l'histoire des Juifs d'Allemagne. Toutes les stations de la vie de Martin Buber, Vienne, Berlin ou Jérusalem s'accompagnent de remarques d'une grande pertinence sur la population et l'état de développement de la ville à l'époque considérée.

Dans son introduction, Bourel souligne que Buber est une figure mal connue, et souvent mal jugée en France et l'un des objectifs avoués de sa biographie est de tenter d'y remédier (p. 16). Comme toutes les grandes biographies, celle que livre Dominique Bourel est bien plus que cela. Elle éclaire l'œuvre de Buber à la lumière de sa vie, prend en compte les contextes politiques, intellectuels et culturels dans lesquels elle s'inscrit et montre Martin Buber comme un homme engagé dans son siècle. Le lecteur y découvre Martin Buber, à la pointe de tous les courants intellectuels et au cour des débats de son époque.

Bourel réinscrit l'auteur dans sa généalogie, qui le lie intimement à l'histoire des Juifs dans l'empire austro-hongrois, puis par la suite à toute l'histoire des Juifs d'Europe au XXe siècle. On ne peut naturellement pas évoquer tous les aspects de ce livre aussi foisonnant que la vie et le caractère de celui qui en est l'objet, quelques points saillants méritent néanmoins d'être notés. Bourel revient sur la "Renaissance juive», ce mouvement de remise en cause de l'assimilation par les populations juives dans la période de Weimar. Cela s'exprime chez Buber en réaction à un point de vue, dont le philosophe Hermann Cohen est considéré comme la figure de proue (p. 228). Cette volonté de redécouvrir la culture juive, qui émerge entre les deux guerres n'est pas l'apanage des représentants du mouvement sioniste, mais est partagée par des penseurs comme Franz Rosenzweig, qui défend la légitimité de la vie juive en diaspora et en appelle, sous le nom de "dissimilation", à un retour à la tradition juive. Buber est aussi pour beaucoup synonyme de ce qu'il est convenu d'appeler la philosophie du dialogue, une pensée du langage qui place en son cour la parole parlée et celui qui la profère. Et c'est un aspect qui est très largement traité par Dominique Bourel dans sa biographie. Il traite bien entendu de l'«œuvre majeure» de Buber, intitulée Je et Tu, dont il évoque à la fois, les sources, la réception et les implications philosophiques.

Mais Bourel montre également à quel point le dialogue pris dans son sens concret est au cour de la vie et de l'action de Martin Buber. Il participe notamment à l'élaboration de la revue Die Kreatur (p. 367-369), qui constituait une expérience de dialogue judéochrétien vécu concrètement. Il s'agissait d'un organe, paru entre 1926 et 1930, et dont la publication était coordonnée par le catholique Josef Wittig, le protestant Viktor von Weizsäcker et le Juif Martin Buber. Bourel évoque aussi longuement le rôle déterminant que Martin Buber joue dans la redécouverte dont font l'objet les Juifs d'Europe 
orientale dans l'entre-deux-guerres. Les histoires hassidiques qu'il retrouve, édite et remet au goût du jour à partir de 1908 ont marqué l'imaginaire de l'ensemble d'une génération et est une des pierres angulaires de la "Renaissance juive» (p. 131 sq.).

Mais un des grands mérites de cette biographie est de montrer que c'est dans toute l'Europe, voire dans le monde entier que Buber nourrit des contacts, pratique le dialogue. Il est notamment en contact après la Seconde Guerre mondiale avec de nombreux intellectuels français. On citera entre autres la revue Esprit, ou les Études méditerranéennes - il participe à deux reprises au congrès méditerranéen, organisé par Joe Golan, fondateur des Études méditerranéennes (p. 642 sq.). Dominique Bourel qualifie Buber de "Juif universel», et il est frappant de constater qu'après-guerre, il n'a de cesse de nouer et entretenir des contacts avec des intellectuels de tous pays et d'cuvrer pour la paix, la liberté et la fraternité entre les peuples. Il s'engage notamment contre l'apartheid en Afrique du Sud, ce qui paraît pour le moins inattendu (p. 617). Bourel rend également compte d'une rencontre avec le philosophe Martin Heidegger en mai 1957 (p. 616).

Cette biographie donne enfin l'image d'un Buber visionnaire dans sa manière d'envisager le sionisme, puis la création de l'État d'Israël et comme un homme qui a eu à coeur de prendre en compte et d'apprendre à connaître les populations arabes. II constitue la preuve vivante que le soutien au projet sioniste n'était pas nécessairement incompatible avec le respect des populations installées en Palestine avant 1948 et comment le conflit judéo-arabe s'inscrit peu à peu, mais de manière toujours plus irrémédiable dans l'histoire de l'État hébreu.

L'index, les notices biographiques et la bibliographie aident le lecteur à s'orienter et lui permettent au besoin d'approfondir certains points. Étant donné l'ampleur de l'ouvrage, on pardonnera aisément quelques imprécisions dans les notes, où manque parfois la référence au numéro de page. Le choix des titres et des intertitres est manifestement important pour l'auteur qui y a mis beaucoup d'ingéniosité et souvent beaucoup d'humour. Ces derniers sont cependant suffisamment transparents pour permettre un ciblage de thèmes précis et une lecture sélective de certains passages. On signalera également les très beaux documents iconographiques, qui occupent le centre de l'ouvrage et montrent Buber, avec sa famille, ses amis et ses collègues. Ils contribuent à donner à cette grande figure un caractère à la fois vivant et emprunt d'émotion.

Mélange d'érudition et d'anecdotes parfois truculentes, mais toujours éclairantes sur la vie de Martin Buber lui-même ou sur son époque, les personnalités qu'il a rencontrées, le tout appuyé sur un appareil de notes parfois vertigineux, la biographie que livre Dominique Bourel n'est pas seulement un livre à la fois très informatif et plaisant, elle est un véritable tremplin pour la recherche, on y trouve en effet des pistes prometteuses pour des travaux à venir. 